\title{
Structural Awareness for Collaborative Learning Environments
}

\author{
Pablo Reyes ${ }^{1}$ and Pierre Tchounikine ${ }^{2}$ \\ 1 Universidad de Valparaíso, Faculty of Sciences. \\ Avda. Gran Bretaña 1111 Valparaíso, Chile. pablo.reyes@uv.cl. \\ 2 Université du Maine, LIUM-CNRS FRE 2730 \\ Av. Olivier Messiaen, 72085 Le Mans, France. \\ pierre.tchounikine@lium.univ-lemans.fr
}

\begin{abstract}
In this paper we propose a peer-to-peer support approach that we call structural awareness support. The structural awareness aims to support the communication that takes place in virtual learning communities. Its emphasis is on revealing the group structure to its participants in order to promote collaborative interactions. This support has been implemented on a forum type tool called Mailgroup. It has been tested twice in different contexts, obtaining initial feedback of its pertinence according to our objectives.
\end{abstract}

\section{Introduction}

Network technologies led to the establishment of new web-based learning activities and the emergence of new types of communities called virtual learning communities (VLCs). The VLC expression is used in this paper to designate online social systems, where their actors are generally geographically distributed, collaborate usually via ICTs, and maintain a shared purpose [10].

Particularly, we focus our research on providing mechanisms to support VLCs. In VLCs, collaborative learning activities are mainly carried out through a conversational, written and asynchronous environment, which we call forum-type tools. The term "forum-type tools" (FTTs) is used for text-based and asynchronous electronic conferencing systems that make use of a tree hierarchical data structure of chained messages called threads. In their origin, FTTs were designed for the distribution of news, not as an environment for interactive communication [5]. Although research has shown some difficulties associated with FTTs for educational use $[6,7,18]$, these tools are currently widely used in this domain (computer conferencing packages, web-based newsgroups and e-learning platforms). In this 
context, we address issues related to the structure of learning conversations taking place in VLCs. Particularly, our research issue is to analyze learning conversations taking place in VLCs in order to provide mechanisms to support and facilitate the emergence of these interactions among the users of FTTs.

In order to create this support mechanism, we work with a methodology we call "technological innovation". The tenet of this inductive methodology is that the design of new technologies deals with "revealing anomalies in practices". An anomaly is an invisible tension that exists when users carry out a particular activity, which for phenomenological reasons people are sometimes unaware of. Nevertheless, they are found at the origin of work practice dissatisfactions. Thus, we create new technologies that aim to overcome the found anomalies.

This paper is organized as follows. First, we present the anomalies found from a relational perspective and their associated incongruencies. Next, we describe the structural awareness perspective that aims to overcome the anomalies found. Finally, we present our prototype, which is built over these propositions and we present some results from an empirical study.

\section{Anomalies in VLCs}

We can analyze the conversations and interactions that take place in VLCs that use FTTs from different points of view (semantically, sociologically, pragmatically, etc). In this work, we understand and analyze interactions from a relational perspective. In this sense, we no longer interpret the conversations as semantic chains of participants' contributions, but take an approach where we interpret interactions in a purely relational way. That is, we think about interactions just as links between messages in conversations. In the case of an FTT, the link is produced by an explicit action of a participant that relates the messages by answering a preceding message or by creating a new one. From the study of these interactions (in a relational perspective) and the network of relations that generate them, we wish to reveal certain anomalies in the communication that takes place in FTTs.

We conjecture that if we can identify what we call structural incongruencies, anomalies become visible. Structural incongruence is a difference between the expression of the users' actions and the perception of the structure of interactions that turns out to be a salient product of these actions in the current FTT. Therefore, in this work we look for structural incongruencies as a way to make anomalies salient. We have used different methods for analyzing data to reveal anomalies (observation analysis and quantitative analysis).

Observation analysis took place principally in a selection of USENET newsgroups (comp.ai.philosophy, humanities.lit.authors.shakespeare, humanities. philosophy.objectivism, sci.anthropology.paleo, soc.culture.french, talk.origins, talk.philosophy. humanism, talk.politics.guns.). This option is not in opposition to our aim of studying VLCs. Research has shown that some newsgroups can be considered as a community [14]. The quantitative studies are based on the analysis of FTT interactions of a set of eight selected newsgroups (the most active and 
having longer threads length) in order to unveil evidence of anomalies based on solid data from real usage.

In our study of FTTs we found four different incongruencies: (1) the interactional incongruence derived from the difference between the message unit that users can handle and the one they can make reference to, causing information loss and reference confusion; (2) the convergent incongruence triggered by the lack of a mechanism to manage the convergent interactions taking place in FTTs, such as consensus, connecting ideas or making a synthesis; (3) the turn-taking incongruence caused by the disruption between (a) the temporal and thread order of messages, and (b) the management of parallel threads; and (4) the group perception incongruence caused by the deficient perception of the interaction structural regularities taking place in a group.

Interactional Incongruence: It has been detected that within a message we can find several topics that appear and develop as threads [1, 18]. Users usually answer by choosing a fragment of the message that they want to answer. This segmentation is done by (a) selecting a part of the message, or (b) selecting several parts of it when answering several paragraphs. However, the segmentation is not visible in the current implementation of systems based on message threads (that we call interactional incongruence), since this only denotes the link relation between messages and does not take into account the specific parts selected by users. Consequently, when examining a discussion through the current tools, topics are not easily localized because they are buried in the rest of the message content. This incongruence is detailed in [12].

Convergent Incongruence: Collaborative learning activities include "divergent interactions" such as brainstorming, which is a highly generative and dispersed interaction that does not tend to build collective results and "convergent interactions" such as the process of synthesizing, summarizing, reaching a consensus, and connecting or integrating different ideas. Moreover, different authors have observed the lack of mechanisms for convergence of FTTs $[7,12]$. In an empirical study, Rocco [15] finds that consensus convergence and social enforcement were weaker in a FTT than in a face-to-face context.

The lack of convergence can be partially related to the intrinsically divergent representations used in FTTs [7]. The data structure of threaded conversations is a tree structure that diverges naturally on multiple branches from the top-level message (initial message). Even if the messages convey synthesis, consensus, or other convergent interactions among different branches of a thread, these cannot be visualized on the data structure, and consequently, they are "buried" among all the messages. This situation is named convergent incongruence.

Turn-taking Incongruence: By turn-taking incongruence, we refer to related issues in the management of turn-taking in FTTs. This incongruence has serious consequences for the emergence of learning conversations. The turn-taking in spoken conversations is a process that takes place in an orderly fashion. In each turn the participant speaks and then the other responds, and so on. Thus conversations are oriented as a series of successive and negotiated steps or turns, so turn-taking becomes the basic mechanism of conversation organization. The turn-taking system in $\mathrm{CMC}$ tools is substantially different from face-to-face communication (e.g., [8, 9]). The communication carried out using these tools follows a multidimensional 
sequential pattern (through parallel threaded conversations), rather than a linear sequential pattern, with "complex interactions that result in layered topics, multiple speech acts, and interleaved turns".

We conjecture that the lack of linearity and the dislocation of turn-taking are related through two dissociations. First, the dissociation in threaded conversations between the temporal and the thread order of messages (Figure 1). Second, the dissociation between a particular work pattern [8] (users do not send messages in a regular frequency, they answer generally in a buffer-like way to different threads) and its representation on FTTs. The importance of orderly turn-taking can be found in [16], which observes that through iterative turn-taking structures, "students are able to build on each other's ideas and intentions, draw new ideas into a common conceptual frame, and repair divergences" [16].

Regarding the first dissociation, in threaded conversations, several authors have observed a dissociation between two possible views in current FTTs: the temporal and the thread order of messages $[2,6]$. This dissociation is caused by the dislocation of the factors of space and time in most of the threaded conversation visualizations. In FTTs, users are able to look at the same conversation from two different viewpoints: messages ordered by time or by threads (Figure 1). This dislocation makes conversations not as seamless as they should be: sorting by threads makes the tracking of timely exchanges difficult. Along these lines, Davis and Rouzie [2] note "as messages can be added to any node in a thread at any later date, students often failed to follow the development of a particular debate" [2, p.12]. But sorting messages by time does not allow users to regard the actual placement of a message in the threads.

Regarding the second dissociation, some authors have observed that users have a particular work pattern [8]: users do not send messages in a regular frequency, but tend to answer in a buffer-like way. They successively concentrate their replying activity on sending several messages in a short period of time in order to bring their interventions in a conversation up to date (consecutive messages). In these responses they sometimes answer two or more consecutive messages in different threads. In $[13]$ is found the quantitative study that confirms this temporal work practice in some newsgroups.

Group Perception Incongruence: We notice that users in face-to-face interactions are able to perceive structural regularities such as the level of

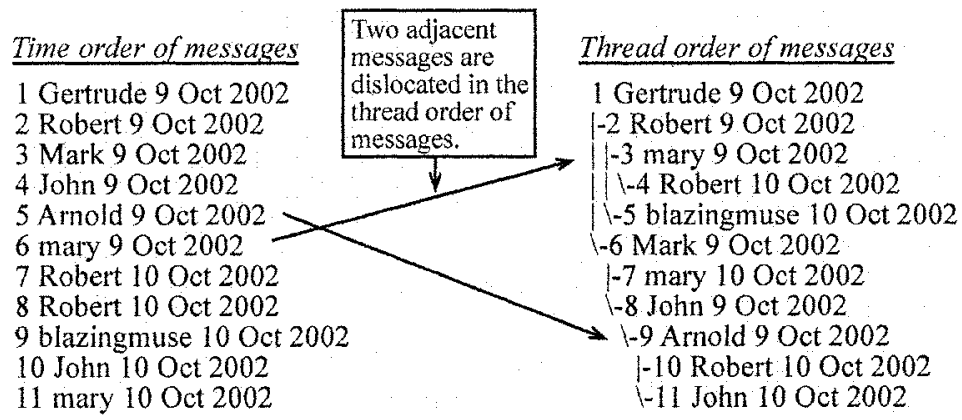

Fig. 1. Dissociation in threaded conversations between the temporal and the thread order of messages. 
interactivity in the group (i.e., if it is talkative or not), its clusterability (whether it has different subgroups), role of participants (e.g., identification of the group leader), as well as others. Nevertheless, for those who communicate through FTTs, it is difficult to perceive these structural properties.

The lack of perception of these structural regularities can be an element that diminishes the social presence of a group. The social presence is a measure of the feeling of community that a participant experiences in a virtual environment [17] and this corresponds to the degree of awareness of a participant in his or her interactions with others and their consequent appreciation of the interpersonal relationships that are constructed from these interactions. Gunawardena [3] indicates that the social presence is needed to improve learning.

\section{Structural Awareness}

We propose a peer-to-peer support approach called structural awareness as a way of overcoming the detailed incongruencies. In this section, we explain the notion of peer-to-peer support and describe the structural awareness support perspective.

\subsection{Peer-to-peer Support}

The kind and extent of support mechanisms provided to the users by the CSCL platforms are a critical element in the design and conceptualization of these educational tools. These support mechanisms are a very active field of research in educational technologies. Multiple strategies and developments exist in this field. However, in this multiplicity of support initiatives in collaborative learning, we can distinguish three possible approaches: "teacher-supports-students", "automatedsystem-supports-students" and "students-support-students". The main difference between these approaches lies on the locus of processing who provides the support.

The students-support-students approach, what we call peer-to-peer support, has as a characteristic element the reciprocal assistance and organization between participants. Peer-to-peer support is a bottom-up approach: One does not seek to make a system that intervenes on the actors, but a system that gives them the means of intervening by themselves. Consequently, the strategy of support is based on the need to inform the actors, enabling them to be conscious about their activity.

\subsection{The Structural Awareness Design}

Structural awareness deals with knowledge about structural features or activities of a group. It puts emphasis on revealing the structural properties of a group to its members in static and dynamic viewpoints in order to promote better collaborative interactions. Structural awareness is a strategy to overcome the anomalies detailed in the previous section.

This option is mainly based on two ideas. First, we are interested in studying and supporting the peer-to-peer learning perspective. Moreover, we think that there is 
insufficient research on this peer-to-peer approach in the domain of supporting interactions. Secondly, looking at the structural perspective of interactions, we see that it is motivated by the relational perspective of conversations taken in this work. Moreover, we think that there is a lack of research on structural perspectives as applied to the domain of supporting interactions.

The foundational idea of structural awareness support is making salient to users some structural aspects, features and anomalies of the group. With the structural awareness support we do not seek to build a system that intervenes on the actors, but a system that gives them the means for intervening by themselves. The basis for this idea is that a proper knowledge of the interactions structure helps users to be conscious about their activity, and by being conscious they obtain benefits to the self-management of interactions.

Structural awareness employs different strategies that aim to make more coherent the VLC users' actions and perceptions of these actions that take place in FTTs, overcoming the identified incongruencies. These strategies try to facilitate the emergence of learning conversations. In particular, the structural awareness will be reified through different strategies that aim to enable participants to perceive their interactions in what we suggest is a more congruent and concise way than in current FTTs, by being able to see the structure of their exchanges in an ordered visual manner, thus overcoming the interactional, convergence, and turn-taking incongruence already mentioned. Thus, we show the participants the group's structure of interactions, enabling them to perceive their interactions through a graph-like visualization, providing particular mechanisms for maintaining a higher coherence between their interactions and the visualization. Additional reification occurs through a set of persistent indicators of certain characteristics of the group, which allows users to get summarized information related to some group structural properties, and even some structural attributes of individuals, that are not salient in VLCs based on some quantitative indicators, namely the complexity of a group, cohesion of a group, and individual status indicators.

All of these strategies aim, according to our structural perspective, to show different aspects of the group structure of interactions, in order to help users to perceive their interactions from this perspective. We conjecture that this perspective can give users new elements for their peer-to-peer support of learning conversations that take place in VLCs. All these strategies have been implemented in an experimental FTT tool we call Mailgroup.

\subsection{Proposed strategies}

We propose several strategies that make up the structural awareness support. Each of these strategies, which are implemented on the Mailgroup FTT, aims to overcome an identified anomaly.

Regarding interactional incongruence, we propose changing the current minimal unit of exchange (the message) for a new minimal unit, the topic. The topic is a subset of a message, generally a smaller portion of it, which has been selected by a user. This change will allow us to make reference to any segment of the message and thus easily track every participant's intervention throughout his interactions. The localization is carried out through the What You Answer is What You Link (WYAIWYL) principle [12]. Therefore the participants, in order to respond to a 
specific topic, must explicitly select it. We note that the topic is not an "object" previously defined (a topic is only a part of a message), but becomes a visible object once it is selected through the WYAIWYL principle.

We propose a tool that allows users to create a link between the selected topic and the answer. Therefore, threaded conversations can be defined by the users from the selected topics. It is important to note that we do not use any semantic text analysis method for locating the topics: They are defined by the participants. Thus, the WYAIWYL principle allows users to define their threads freely, thereby overcoming the interactional incongruence. This strategy is detailed in [12].

Regarding turn-taking incongruence, we propose a visualization of threads, so one can quickly and graphically visualize the flow and patterns of online discourses at the same time. We will merge both types of existing views (the time order and the thread order of messages) in a single view that graphically depicts both concepts, as presented in the next section. Moreover, we propose the creation of a structure called session that aims to overcome temporal incongruence. This structure intends to model the turn-taking behavior and make the particular rhythm of answers visible. A session corresponds to a group of messages sent by the same participant consecutively in a short period of time. In other words, it is a new structure that holds together the messages sent almost at the same time.

This structure corresponds to columns (Figure 2) that package the messages in a parallel and linear way. These arrangements seek to reduce the dispersion and the dislocation of threaded conversations by augmenting the linearity of conversations and allowing one to start or to maintain parallel discussions in a unified visualization. This strategy is detailed in [13].

These two strategies (merging time order with the thread order of messages, and the integration of session construct on a FTT) seek to reduce the dispersion and dislocation of threaded conversations, by augmenting their linearity and making it possible to start or maintain parallel discussions in a unified visualization.

Regarding convergence incongruence, we propose a new functionality that

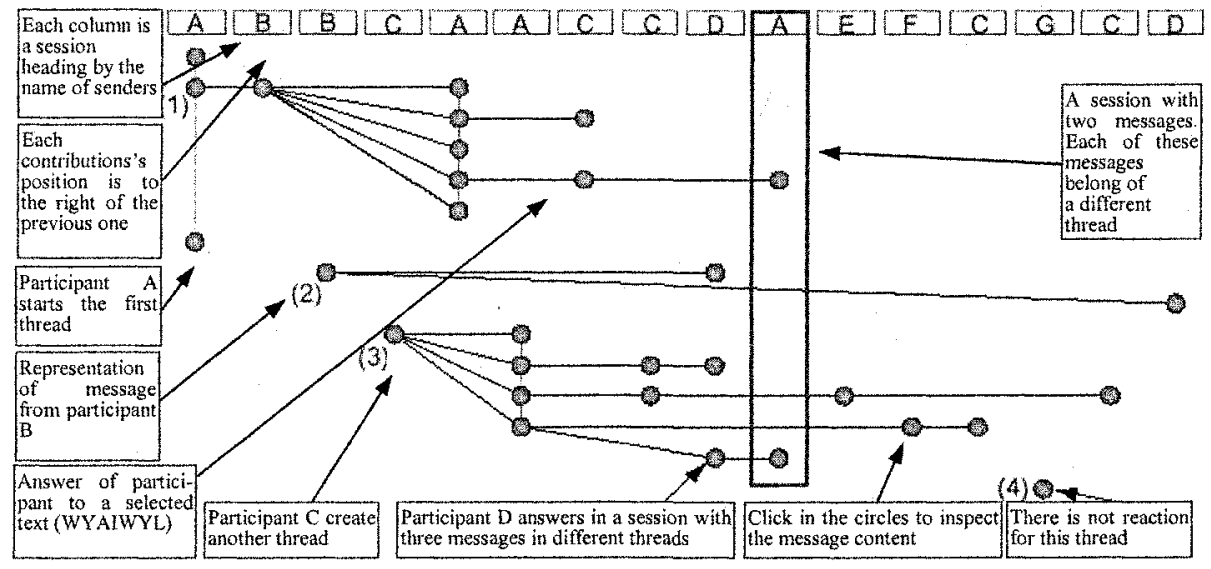

Figure 2. "TIC and disciplines" conversation in Mailgroup

allows multiple threads to merge into a single one. The result of this operation is 
properly visualized through the visual space in the proposed tool. Consequently, all users will be aware of the existence of convergent interactions and also they can inspect the convergent interactions and react to them.

Regarding the group perception incongruence, we propose the creation of an observation level which allows users to get summarized information related to some structural properties that are not salient in VLCs. Here, we have implemented an observation level of the communicational activity based on quantitative indicators (complexity, cohesion, and status indicators). This level offers some synthesized group behavior information about users, which is neither easy nor transparent to obtain from the level where the message interactions actually take place in an asynchronous and text based communication.

\section{Tests}

Empirical studies were designed in order to collect feedback on the actual characteristics of our prototype from the user's perspective. During this study, the tool was used just as the medium of communication, not as a point of concern in itself. We did not want the students to discuss the tool, nor think about it. We were interested in evaluating just how they could "work it out". We tested Mailgroup in order to: (1) test the system usability and (2) obtain the first corpus that allows us to analyze how Mailgroup is used and what is going on when people use this system.

\subsection{Empirical Context}

Mailgroup has been tested twice. First, a test that aimed to get the feeling of users towards the proposed tool. In this study, nine groups of 3 or 4 students (32 participants in total) were recruited. They were second year undergraduate students, who voluntarily carried out, for one month, a distance collaborative activity. Second, a test with a deeper and more complete usability study and corpus analysis. In the second test, 15 participants were recruited. All of them were teachers, who for a month and a half carried out a distance collaborative activity as part of a training course on information and communication technologies.

\subsection{Results}

First, we detail one conversation that took place in Mailgroup. Next, we present some results of the survey. Finally, we present the principal findings found through the realized tests.

Figure 2 shows the "disciplines" conversation. Here seven people took part and four threads were created by four different members. The initial number of threads of this discussion (4) can be considered initially as low. But the exchanges are very rich. They include recommendations, advice, and examples proposed to others. There are also interrogations and incentives to offer an opinion. Moreover the thread depth (i.e., the number of responses within a particular thread) goes up to five levels. 
Consequently, the group does not stay in a situation of question and answer. In total, when regrouping the messages, this conversation constituted a document of almost 3 pages.

In order to obtain the students' impressions of the use of Mailgroup as a communication tool, we conducted a survey of the participants on the last day of the training course. In general, a high percentage of participants (75\%) considered that the proposed visualization and organization of messages allow for a better following up of the ongoing development of conversations. Moreover, a high percentage of participants $(75 \%)$ considered that Mailgroup permits an effective visualization of the participant's exchanges. Only a small percentage (13\%) had difficulties following up threads in the proposed visualization.

Participants used the WYAIWYL principle. Since students could choose more granular and "findable" topics in the easily navigable thread view, they could organize and collaborate better. Also, the most significant benefits of using this criterion identified by users were: (a) an easier identification of the active threads and (b) it better allowed for the organization of answering, relative to current FTTs.

Consequently, the survey, though limited, seems to strongly show the benefits of using this visualization for threaded conversations. The results of this study are preliminary, mainly due to the relatively small size of the sample. Nevertheless, the results have allowed us to obtain good feedback about the usability of the proposed system. These results indicate that the proposed system and the concepts underlying the construction of Mailgroup are significant.

\section{Conclusions}

We propose structural awareness support. This support aims at users being aware of their activity through their communication structure. The tool presented in this paper focuses on the notion of enhancing coherence in threaded conversation systems. It is aimed at facilitating the emergence and development of learning conversations. This was done overcoming some incongruencies we have identified as undermining the emergence and development of better communication.

Our principal findings are as follows. (1) The experience carried out with Mailgroup confirms that WYAIWYL principle is a useful mechanism for structuring FTT threaded conversations. (2) The use of this tool enables a change of practice: students organize the structure of messages into paragraphs. Indeed, they affirm that creating messages in this way will make it easier for other participants to locate and respond to parts of a message (topic), facilitating topic salience and definition. (3) Another observation is the salience of no-answer topics. The proposed visualization facilitates the tracking of no-answer topics that take place during the conversation. These topics are visualized and highlighted in the interface as circles without links to or from other messages. (4) Indicators can be an element used to analyze the group in order to obtain a structural awareness of their interactions, although more studies concerning this must be made.

We consider that in future developments the graph visualization must be improved by (1) using algorithms that allow fewer lines to be crossed between 
messages, because these lines make the visualization of references between messages difficult; and (2) optimizing the space that is now poorly exploited (in the current visualization there are many zones without messages).

\section{References}

1. Condon, S. L., \& Cech, C. G. (Forthcoming). Discourse Management in Three Modalities. In S. Herring (Ed.), Computer-Mediated Conversation: Hampton Press.

2. Davis, M. and A. Rouzie (2002). Cooperation vs. Deliberation: Computer Mediated Conferencing and the Problem of Argument in International Distance Education. International Review of Research in Open and Distance Learning 3(1).

3. Gunawardena, C. N. (1995). Social presence theory and implications for interaction and collaborative learning in computer conferences. International Journal of Educational Telecommunications. 1: 147-166.

4. Gutwin, C., Greenberg, S., \& Roseman, M. (1996). Workspace Awareness in Real-Time Distributed Groupware: Framework, Widgets and Evaluation. Paper presented at the HCI 1996.

5. Hauben, M., \& Hauben, R. (1997). Netizens: On the History and Impact of Usenet and the Internet: Wiley-IEEE Computer Society Press.

6. Herring, S. (1999). Interactional Coherence in CMC. Paper presented at the 32nd Hawai'i International Conference on System Sciences, Hawai.

7. Hewitt, J. (2001). Beyond threaded discourse. International Journal of Educational Telecommunications, 3(7), 207-221.

8. McElhearn, K. (2000). Writing conversation : an analysis of speech events en e-mail mailing lists. Revue Française De Linguistique Appliquée 5(1).

9. McKinlay, A., R. Procter, et al. (1994). Studies of turn-taking in computer-mediated communications. Interacting with Computers 6(2).

10. [Mowshowitz, A. (1997). Virtual Organization. Communications of the ACM, 40(9), 3037.

11. [Pincas, A. (2001). E-learning by virtual replication of classroom methodology. Paper presented at the The Humanities and Arts higher education Network, HAN.

12. [Reyes, P., \& Tchounikine, P. (2003). Supporting Emergence Of Threaded Learning Conversations Through Augmenting Interactional And Sequential Coherence. Paper presented at the CSCL Conference.

13. [Reyes, P., \& Tchounikine, P. (2004). Redefining the turn-taking notion in mediated communication of virtual learning communities. Paper presented at the Intelligent Tutoring Systems, Maceió, Brazil.

14. [Roberts, T. L. (1998). Are newsgroups virtual communities? Paper presented at the CHI'98.

15. [Rocco, E. (1996). Cooperative efforts in electronic contexts. Americas Conference on Information Systems.

16. [Roschelle, J. (1992). Learning by collaborating: Convergent conceptual change. Journal of the Learning Sciences 2(3): 235-276.

17. [Tu, C.-H. and M. McIsaac (2002). The relationship of social presence and interaction in online classes. The American Journal Of Distance Education 16(3).

18. [Vapillon, J. (2000). Contribution à l'étude de la conversation dans le cadre du travail coopératif assisté par ordinateur. Paris XI University, Paris. 